\title{
CRYPTEL - THE PRACTICAL PROTECTION OF AN EXISTING ELECTRONIC MAIL SYSTEM
}

\author{
Hedwig Cnudde
}

CRYPTECH NV/SA

Project Management

B-1050 Brussels, Belgium

\section{ABSTRACT}

This paper describes the practical protection of an existing Electronic Mail System using the RSA-algorithm.

\section{INTRODUCTION}

BISTEL, which stands for "Belgian Information System by Telephone", is an office automation system set up by the Belgian Government. This system aims at making the access to information and its treatment more efficient and easier.

BISTEL, operational since 1984 combines wordprocessing systems, communication systems and systems for consulting data bases.

The present system consists of a central host computer and terminals spread over the various ministerial departments. Each terminal is connected to the central host computer through the public switched telephone network, the public X.25network or the telex-network. 
As a part of BISTEL, a system of Electronic Mail using ordinary terminals together with PC's as Computer Based Terminals allows the users to communicate with each other, in all possible ways used for day-to-day correspondence; this system can also be applied for sending documents such as the agendas and the documents for the Council of Ministers and for Ministerial Committees.

It was decided to secure the Electronic Mail in the BISTEL-system. This project has been called CRYPTEL (CRYPtography bisTEL). INTERSYS (today CIG-Intersys), the main contractor for the BISTEL-system, assigned this job to CRYPTECH, a company specialized in data security systems.

CRYPTECH is not only the Main Contractor of the CRYPTEL project but also developed most of the required equipment.

\section{THE USE OF RSA}

A preliminary study ([1]) by the ESAT laboratory of the 'Katholieke Universiteit Leuven' showed that a public key crypto-system (based on the well-know RSAalgorithm of Rivest, Shamir and Adleman) would be the best choice for the protection of the Electronic Mail in the BISTEL-system. This algorithm lends itself very well to guarantee the privacy of a message as well as to verify the integrity of the message and the authenticity of the sender.

The RSA-algorithm (used with a key length of 672 bit) implies the manipulation of blocks of about 80 characters.

\section{SEVERAL ENCRYPTION/DECRYPTION RUNS}

A distinction must be made between:

- Communication between 2 correspondents.

- Communication between the host computer and a correspondent. 
The former communication is protected by means of end-to-end encryption, while the latter is protected by link encryption. Starting from this distinction one chooses a combination of encryptions:

- The first operation is a double encryption of a message which will only be decrypted by the correspondent. This allows for both privacy and authenticity protection of the original message.

- The second operation is a double encryption of the communication with the host computer. As such the privacy and the authenticity of this communication are being guaranteed.

Special practical problems that had to be solved, were:

- privacy and authenticity protection of messages by means of RSA;

- verifying the authenticity of people and equipment by means of RSA;

- secure storage of RSA keys using a Smart Card;

- authenticity protection of public keys;

- distribution of public keys;

- local file ecncryption.

\section{THE USE OF PC'S}

When using RSA, all characters are manipulated and transmitted in blocks of a certain length. Thus, characters are buffered until a block is filled or until a transmit signal is given.

Use of cryptography has been restricted to Computer Based Terminals (say PC's). Eventually these may be equipped with a Word Processor to allow for the local editing of a message to be transmitted. Anyhow, without any doubt, a PC based solution offers many more advantages: 
. PC's are the workstations of the future because of their flexibility;

- the use of PC's offers many more possibilities than just securing Electronic Mail: e.g. files can be stored locally in an encrypted form;

- these PC's can easily be reused in other secure systems.

\section{AT THE TERMINAL'S SIDE: THE PC-RSA SECURITY CARD}

A software implementation of the RSA-algorithm is considered to be much slower than a hardware version. Since the algorithm must be executed on-line and very quickly, a hardware implementation has been chosen. As a consequence, a special RSA-chip has been developed by CRYPTECH.

The PC-RSA Security Card, a microprocessor based board, contains an RSA chip-set together with control logic and firmware as well as all communication and key loading interfaces.

So, for the crypto-unit in the Computer Based Terminal (i.e. a PC), one opted for a built-in single-board system (occupying 1 slot) connected to an input device for the cryptographical keys: The PC-RSA Security Card.

\section{SECURITY PHILOSOPHY REGARDING THE NETWORK}

For each type of transmission, the following rule of thumb can be used: "The encryption of a message (text or control) can be executed at that very place where the information should no longer be present in clear".

Analogously the data must be decrypted at the other side there were the controller text must be readable again. E.g., the headers of the packets of the X.25 protocol can not be encrypted unless one moves the encryption to the network which again means a lowering of the security. 
The encryptions and decryptions should preferably be done as close as possible to the user.

\section{AT THE HOST'S SIDE: THE BULK-ENCRYPTOR}

The same hardware as in the Computer Based Terminal, in a more complex form, can be used at the level of the host computer:

- This device makes sure that the information intended for the central computer is converted to plain text or that the outgoing information is being encrypted.

- The real contents of a document remains authenticated and encrypted for storage and is only being converted into plain by the end user.

\section{VIIITHE KEY MANAGEMENT: CRYPTO-ADMINISTRATOR AND SMART CARDS}

The key management (generation, distribution, storage, transmission, renewal, ...) is a task which should be kept separated from the rest of the network. Some of these operations are executed on a separate computer: a Crypto-Administrator (i.c. a PC).

Only the required keys are being transferred to the Crypto-Units in question. As the capacity of a simple magnetic card is insufficient, the cryptographical keys are stored, for the user, into a Smart Card. A further advantage of the use of Smart Cards is that they can be applied for Access Control.

So, each PC used as a Computer Based Terminal will be equipped with a Smart Card Reader. The CRYPTEL Bulk-Encryptor will be equipped with a Smart Card Reader for the Smart Card of the operator. 
The CRYPTEL Crypto-Administrator will be equipped with 2 Smart Card Readers:

- one Reader will be used for the Smart Card of the operator,

- the other one for the Smart Card to be personalized.

\section{REFERENCES}

[1] J. Vandewalle, R. Govaerts, W. De Becker and M. Decroos, Definitief rapport betreffende encryptering systeem voor de elektronische postdienst en de dokumenttransmissie van het BISTEL informatika systeem van de Belgische regering, April 1985. 